\title{
AUTOMATIC CHOICE OF THE THRESHOLD OF A GRAIN FILTER VIA GALTON-WATSON TREES. APPLICATION TO GRANITE CRACKS DETECTION
}

\author{
ROMAIN ABRAHAM, MAITINE BERGOUNIOUX, AND PIERRE DEBS
}

\begin{abstract}
The goal of this paper is the presentation of a post-processing method allowing to remove impulse noise in binary images, while preserving thin structures. We use a grain filter as in [5]. We propose a method to automatically determine the required threshold using Galton-Watson processes. We present numerical results and a complete analysis on a synthetic image. We end the numerical section considering a specific application to granite samples crack detection: here we deal with X-tomography images that have been binarized via preprocessing techniques and we want to remove residual impulse noise while keeping cracks and micro-cracks structure.
\end{abstract}

Keywords: Image processing; Galton-Watson; grain filters; cracks. MSC classification: 60J80, 68U10, 94A12

\section{INTRODUCTION}

A challenging issue in imaging processing is the identification of thin structures. It may be blood vessels, leaf veins or streets in a satellite or aerial image. From a mathematical point of view these structures have a zero Lebesgue measure and we have to use the Hausdorff measure instead. From a practical point of view, these structures may be not viewed once the discretization has been done. There are many methods to achieve this goal both from theoretical and numerical points of view (as the morphological skeletonization for example). However, they may be not very satisfactory since the images are very often corrupted with a high level of noise that, in addition, may not be Gaussian.

The goal of this paper is not the presentation of an image processing model that would identify the structures better than the existing ones but rather give a post-processing method allowing to remove any residual noise effect. Therefore, we do not report on thin structures recovering techniques, and do not quote the related works.

The example we have in mind is the recovery of cracks inside a granite block. More precisely, the sample has been imaged ${ }^{1}$ via X-tomography process and we have to deal with a $3 \mathrm{D}$ image whose size is $1287 \times 1287 \times 99$. For more details on the geological context one can refer to $[14,21]$. Figure 1.1 shows a $2 \mathrm{D}$ slice of such a stack. The right hand side image is the result of a pre-processing method that we briefly present in Section 3 without any focus on it; it allows to get rid of contours that are not cracks. However, there is a residual impulse noise that we would like to remove while keeping cracks, especially micro-cracks.

Date: January 10, 2017.

${ }^{1}$ We thank Olivier Rozenbaum, ISTO, Université d'Orléans, CNRS, BRGM 


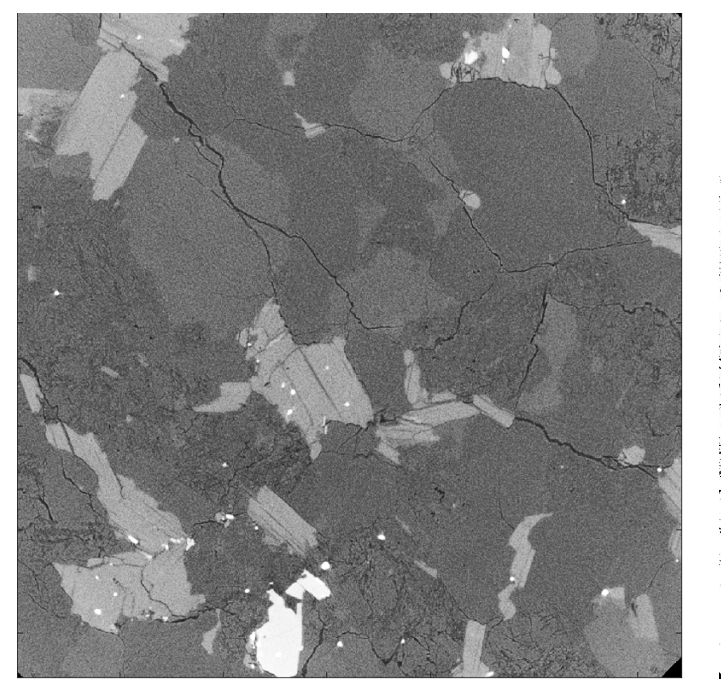

(a) Original Slice

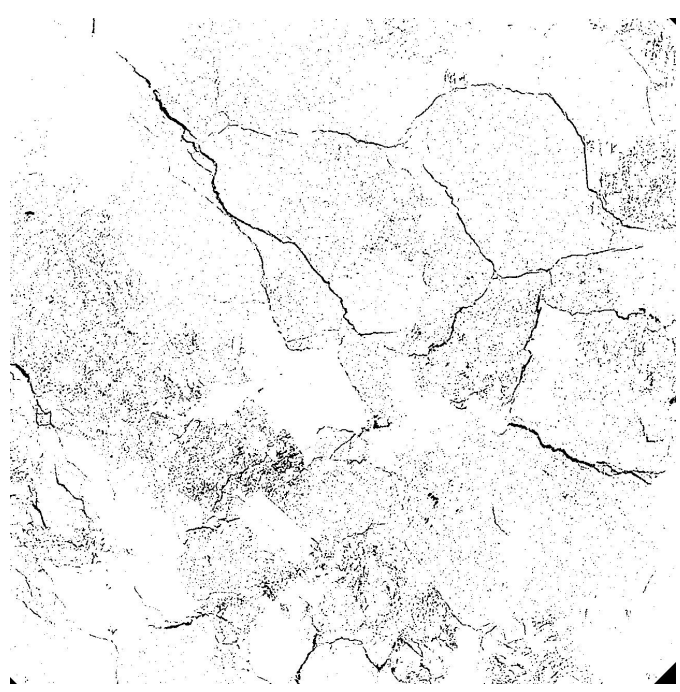

(b) Binarized Slice

FiguRE 1.1. Original and binarized 2D slice of a 3D granite sample

There are few methods devoted to crack identification in geological context. In biological context (angiography or X-ray imaging for example) mathematical morphology (skeletonization) is often used. On can mention the use of fuzzy measures in [12] as well. In material context, there are many works that focus on cracks in roads or concrete. Different tools are used as statistical ones (co-occurence matrices in [15] and anisotropic diffusion with region linkage in [17]), wavelets [23], geodesic contours [6], multiscale approach[7] together with Markov modeling or image-based percolation models [26] and dealing with brightness and connectivity as in [16].

To address this problem, we focus here on a grain filter. This filter, introduced in [25] in the framework of Mathematical Morphology and then generalized in [13], consists in removing all the connected components of a binary image (or of the level sets for a grey level image) of size smaller than a fixed threshold. It is mainly used for image denoising but also for feature extraction, see [20]. We refer to [5] for more details on this filter. In what follows, we suppose that we have a binary image containing features we want to extract (namely thin structures as cracks). The image is corrupted by an impulse noise where every pixel is 1 with probability $p$ and 0 with probability $1-p$ independently of the others. One feature that allows to distinguish cracks from the noise is that the cracks size is much larger than the connected components (that we define in the sequel) the noise may create. Therefore, a grain filter can be used in order to suppress the small connected components and keep the ones whose size is greater than some threshold $a_{0}$. The aim of this paper is to give a method to compute the threshold of the grain filter via a statistical hypothesis testing. This method enters the large class of a contrario frameworks whose ideas first appeared in [24] and which is now widely used since the pioneer works [27, 9]. We refer to the book [10] for a complete review of these methods. The general idea of the a contrario method is to compute the probability of appearance of the looked after feature in a pure noise image. If this probability is very small (less than some fixed $\varepsilon$ ), the feature is said to be "meaningful" i.e. it cannot appear "accidentally" and thus can be viewed as an important characteristic of the studied image. 
Throughout the paper, we consider that the image contains only an impulse noise with parameter $p$ (hypothesis $\mathcal{H}_{0}$ ). Under $\mathcal{H}_{0}$, we denote by $\mathcal{C}_{0}$ the connected component of the image that contains a given point, say the origin $(0,0)$, and by $\sharp \mathcal{C}_{0}$ its cardinal. We then fix a level $\varepsilon>0$ for the test and find $a_{0}$ such that, under $\mathcal{H}_{0}$,

$$
\mathbb{P}\left(\sharp \mathcal{C}_{0} \geq a_{0}\right) \leq \varepsilon .
$$

Keeping the connected components of size greater than $a_{0}$, we remove most of those due to the noise. The goal of this paper is to get an upper bound for the looked after probability, and hence to automatically compute the threshold $a_{0}$, by comparing the size of $\mathcal{C}_{0}$ with the total population size of a Galton-Watson process.

Let us stress that this problem has already been tackled in [8] where an asymptotic (as the size of the image tends to $+\infty$ ) of the probability $\mathbb{P}\left(\sharp \mathcal{C}_{0} \geq a_{0}\right)$ is obtained. This asymptotic contains a combinatorial term $a_{k}$ (namely the number of connected components one can make with exactly $k$ pixels) which is only known (up to $k=47$ ) for the 4connectivity. Our approach first gives an exact bound of the probability and not an approximation as in [8] (which is not very relevant as the size of our images are quite large and the approximations of [8] are sharp), second allows to use different features to discriminate cracks from noise. In Section 2, we focus on the size of a given connected component for the 8-connectivity and give all the details for the proofs but the ideas can be easily adapted to handle other definitions of connectivity, the same problem for 3D images or to use the diameter of the connected component instead of its size as developed as well.

We present the computation of the threshold in the next section and Section 3 is devoted to the numerical realization (algorithms and numerical experimentation).

\section{Computation of the threshold}

2.1. Notations. If $k<\ell$ are two integers, we denote by $\llbracket k, \ell \rrbracket$ the set $\{n \in \mathbb{Z}, k \leq n \leq \ell\}$. We work with the infinite norm on $\mathbb{Z}^{2}$ i.e. if $x_{1}=\left(i_{1}, j_{1}\right), x_{2}=\left(i_{2}, j_{2}\right)$ are two points of $\mathbb{Z}^{2}$, we set $\left\|x_{1}-x_{2}\right\|=\max \left(\left|i_{1}-i_{2}\right|,\left|j_{1}-j_{2}\right|\right)$.

For $x \in \mathbb{Z}^{2}, \bar{B}(x)$ denotes the closed ball (with respect to the previous norm) centered at $x$ with radius 1 and $B(x)$ is the pointed ball $\bar{B}(x) \backslash\{x\}$. The elements of $B(x)$ are called the neighbors of $x$.

For any finite subset $A$ of $\mathbb{Z}^{2}$, we denote by $\sharp A$ the cardinal of $A$.

Definition 2.1. A set $A \subset \mathbb{Z}^{2}$ is said to be connected if, for every $x, y \in A$, there exists a finite sequence $\left(x_{0}, \ldots, x_{n}\right)$ such that

- $x_{0}=x, x_{n}=y$;

- $\forall i \in \llbracket 0, n \rrbracket, x_{i} \in A$;

- $\forall i \in \llbracket 1, n \rrbracket, x_{i} \in B\left(x_{i-1}\right)$.

We fix a positive integer $N$ and consider (for simplicity) the domain $\Omega=\llbracket-N, N \rrbracket^{2}$. A binary image on $\Omega$ will be an application $X: \Omega \longmapsto\{0,1\}$. In all the paper, we always make the confusion between the binary image $X$ and the set $X^{-1}(1)=\{x \in \Omega, X(x)=1\}$, which is the set of the black pixels. In particular, we talk of the connected components of the image instead of the set $X^{-1}(1)$.

2.2. Galton-Watson process. A Galton-Watson process is a branching stochastic model introduced in 1873 by Francis Galton to describe population growth and is still the object of active research. We refer to [2] for a comprehensive exposition on this topic. We first give a formal definition of this process. 
Definition 2.2. A Galton-Watson process is a stochastic process $\left(Z_{n}\right)_{n \in \mathbb{N}}$ defined as follows: consider a probability measure $\nu$ on the set of non-negative integers (called the offspring distribution) and a family $\left(\xi_{i}^{(n)}, n \geq 0, i \geq 1\right)$ of i.i.d. random variables with distribution $\nu$. Then the process $\left(Z_{n}\right)_{n \in \mathbb{N}}$ is defined recursively by

$$
\left\{\begin{array}{l}
Z_{0}=1, \\
Z_{n+1}=\sum_{i=1}^{Z_{n}} \xi_{i}^{(n)} \quad \text { for } n \geq 0
\end{array}\right.
$$

with the convention that $Z_{n+1}=0$ if $Z_{n}=0$.

To give an intuitive picture of that process $\left(Z_{n}\right)_{n \in \mathbb{N}}$, let us describe it informally as a population evolution model. We start with a single individual at generation 0 . This individual gives birth to a random number $\xi_{1}^{(0)}$ of offspring with distribution $\nu$ which form generation 1. These offspring reproduce according to the same distribution $\nu$ independently of each other and independently of the past and so on. Hence, the variable $Z_{n}$ represents the population size at generation $n$ whereas the variable $\xi_{i}^{(n)}$ represents the number of offspring of the $i$-th individual of the $n$-th generation.

Of course, if the population dies out at some time $n$, then the population is zero for all further time: 0 is called an absorbing state for the process $\left(Z_{n}\right)_{n \in \mathbb{N}}$.

One of the initial questions raised by Francis Galton was the computation of the probability that the population dies out in finite time. This probability is called the extinction probability and is given by

$$
\mathbb{P}\left(\exists n \in \mathbb{N}, Z_{n}=0\right)=\lim _{n \rightarrow+\infty} \mathbb{P}\left(Z_{n}=0\right) .
$$

To avoid trivialities, we suppose that $\nu(0)>0$ (if not, the extinction probability is 0 ) and that $\nu(0)+\nu(1)<1$ (if not, the extinction probability is clearly 1 ).

The answer to the question is given by the following result (see Chapter 1 Section 5 of [2]).

Proposition 2.1. Let $\xi$ be a random variable with distribution $\nu$. We denote by

$$
\mu=\mathbb{E}[\xi]=\sum_{k=0}^{+\infty} k \nu(k)
$$

the mean number of offspring, and by

$$
F(s)=\mathbb{E}\left[s^{\xi}\right]=\sum_{k=0}^{+\infty} \nu(k) s^{k} \text { for } s \in[-1,1],
$$

the generating function of $\nu$.

Then, the extinction probability of the process $\left(Z_{n}\right)_{n \geq 0}$ is given by the smallest nonnegative root $q$ of the equation $s=F(s)$.

In particular, if $\mu \leq 1, q=1$ (extinction arises almost surely) whereas if $\mu>1$, then $q<1$ (the population has a positive probability for living forever).

The offspring distribution $\nu$ and the Galton-Watson process $\left(Z_{n}\right)_{n \geq 0}$ are called subcritical (resp. critical, super-critical) if $\mu<1$ (resp. $\mu=1, \mu>1$ ). 
2.3. Stochastic domination. Let $m$ be the number of neighbors of one pixel. With our definition of $B(x)$, we have $m=8$ but the method remains valid for a general neighborhood. Let $p \in(0,1)$ and $\left(Z_{n}\right)_{n \geq 0}$ a Galton-Watson process with the binomial $\mathscr{B}(m, p)$ distribution as offspring distribution $\nu$, that is, for $0 \leq k \leq m$,

$$
\nu(k)=\left(\begin{array}{c}
m \\
k
\end{array}\right) p^{k}(1-p)^{m-k}
$$

According to Proposition 2.1, in order to obtain a sub-critical Galton-Watson process, we assume that $m p<1$. We denote by $\sharp Z$ the total population size:

$$
\sharp Z=\sum_{n=0}^{+\infty} Z_{n} .
$$

Theorem 2.3. Under $\mathcal{H}_{0}$, the size $\sharp \mathcal{C}_{0}$ of the connected component $\mathcal{C}_{0}$ (and thus of any connected component) is stochastically dominated by $\sharp Z$, that is:

$$
\forall k \in \mathbb{N}, \quad \mathbb{P}\left(\sharp \mathcal{C}_{0} \geq k\right) \leq \mathbb{P}(\sharp Z \geq k) .
$$

Proof. The main ideas of the proof are taken from [18] where the size of a connected component that arises in continuum percolation is compared to the size of a GaltonWatson process with Poisson offspring distribution.

Let $\left(X_{(i, j)},(i, j) \in \mathbb{Z}^{2}\right)$ be a family of i.i.d. Bernoulli random variables with parameter $p$. This models the noise of the image under $\mathcal{H}_{0}$. Remark that if the stochastic domination is true for an infinite image, it is a fortiori true for an image defined on $\llbracket-N, N \rrbracket^{2}$. If $A$ is a subset of $\mathbb{Z}^{2}$, we denote by

$$
X_{A}=\left\{(i, j) \in A, X_{(i, j)}=1\right\} .
$$

Then, $\sharp X_{A}$ is distributed according to a binomial distribution with parameters $p$ and $\sharp A$. Moreover, if $A$ and $B$ are disjoint subsets of $\mathbb{Z}^{2}$, then $X_{A}$ and $X_{B}$ are independent.

In order to label the points of some subset in a unique way, we define a total order (denoted by $\leq$ ) on $\mathbb{Z}^{2}$. Let $x_{1}, x_{2} \in \mathbb{Z}^{2}$ :

- if $\left\|x_{1}\right\|<\left\|x_{2}\right\|$ then we set $x_{1} \leq x_{2}$.

- if $\left\|x_{1}\right\|=\left\|x_{2}\right\|=n$, we label the $8 n$ points of norm $n$ as on Figure 2.1

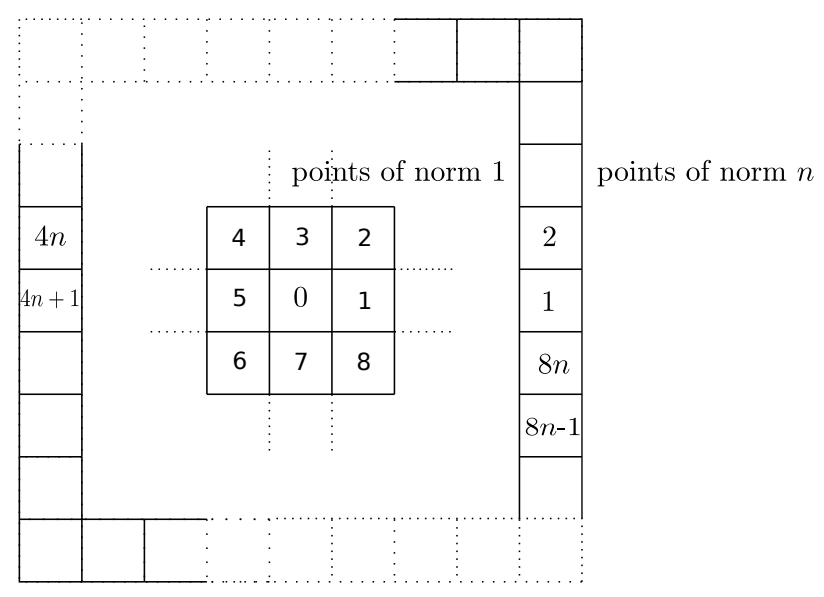

FIGURE 2.1. Labeling of points of norm $n$ 
and we say that $x_{1} \leq x_{2}$ if the label of $x_{1}$ is less than the label of $x_{2}$.

We first construct the connected component $\mathcal{C}_{0}$ recursively. The process is illustrated in Figure 2.2.



(a) Initial state

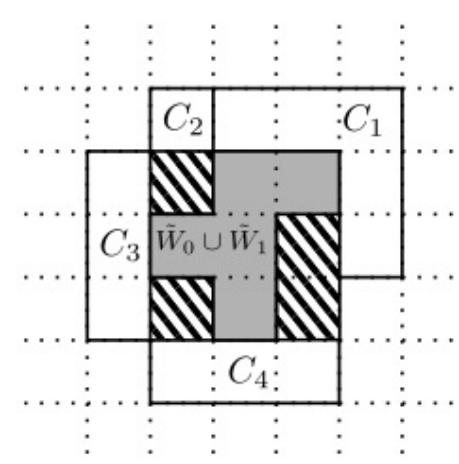

(c) Conditionnally on $\tilde{W}_{0}$, $\tilde{W}_{1}=\{(1,1),(0,1),(-1,0),(0,-1)\}$

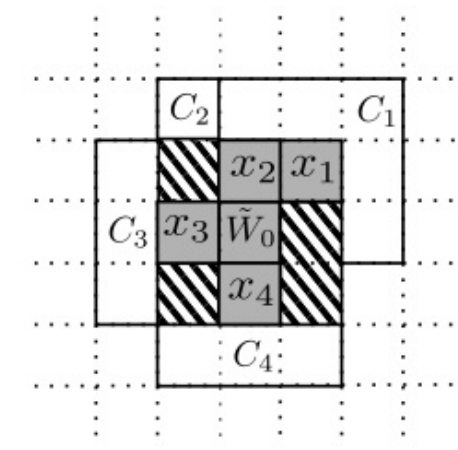

(b) Conditionnally on $\tilde{W}_{0}$, $\tilde{W}_{1}=\{(1,1),(0,1),(-1,0),(0,-1)\}$

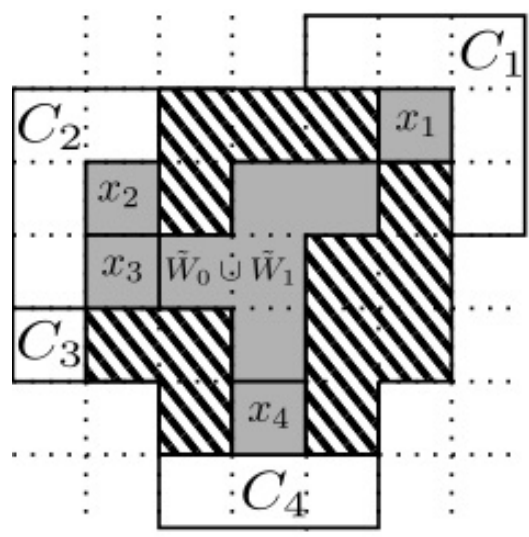

(d) Conditionnally on $\tilde{W}_{0}$,

$\tilde{W}_{1}=\{(1,1),(0,1),(-1,0),(0,-1)\}$,

$\tilde{W}_{2}=\{(2,2),(-2,1),(-2,0),(0,-2)\}$

Figure 2.2. Generation of the sequence $W_{n}$

We define a sequence $\left(\tilde{W}_{n}\right)_{n \geq 0}$ of subsets of $\mathbb{Z}^{2}$ such that $\mathcal{C}_{0}=\bigcup_{n \geq 0} \tilde{W}_{n}$ in the following way:

- $\tilde{W}_{0}=\{(0,0)\}$.

- Suppose that $\tilde{W}_{0}, \ldots, \tilde{W}_{n}$ are constructed with $\tilde{W}_{n}=\left\{x_{1}, \ldots, x_{k}\right\}$ labeled in increasing order. Then, we set

$$
\tilde{W}_{n+1}=\bigcup_{\ell=1}^{k} X_{C_{\ell}}
$$

where

$$
C_{\ell}=B\left(x_{\ell}\right) \backslash\left(\bigcup_{j=1}^{\ell-1} \bar{B}\left(x_{j}\right) \bigcup_{i=0}^{n-1} \bigcup_{x \in \tilde{W}_{i}} \bar{B}(x)\right)
$$


with the convention $\bigcup_{j=1}^{0} \bar{B}\left(x_{j}\right)=\emptyset$.

- If $\tilde{W}_{n}=\emptyset$, then we set $\tilde{W}_{k}=\emptyset$ for every $k>n$.

The set $C_{\ell}$ at step $n$ is the set of neighbors of $x_{\ell}$ that have not been involved yet in one of the $C_{k}$-type set constructed during the previous steps. Consequently, these sets $C_{\ell}$ are pairwise disjoint and the random variables $X_{C_{\ell}}$ are independent and distributed respectively according to a binomial $\mathscr{B}\left(\sharp C_{\ell}, p\right)$ law.

In a second step, we consider a branching random walk $\left(W_{n}\right)_{n \geq 0}$ on $\mathbb{Z}^{2}$ (we refer to [22] for more details on branching random walks). This process takes its values in the set $\bigcup_{n \geq 0}\left(\mathbb{Z}^{2}\right)^{n}$ of finite sequences of points of $\mathbb{Z}^{2}$ (with the convention $\left(\mathbb{Z}^{2}\right)^{0}=\{\emptyset\}$ ) and is also defined recursively as follows:

- $W_{0}=(0,0)$.

- Let us suppose that $W_{n}$ is given and is non-empty, say $W_{n}=\left(x_{1}^{n}, \ldots, x_{k_{n}}^{n}\right)$. For every $i \leq k_{n}$, we consider the 8 neighbors of $x_{i}^{n}$ (ranked as on Figure 2.3) $\left(y_{1}, \ldots, y_{8}\right)$ and consider the sub-sequence $W_{n+1}^{i}$ formed by taking each $y_{j}$ with probability $p$, independently of the others (and independently of the other points $x_{j}^{n}, i \neq j$ ). Then $W_{n+1}$ is the concatenation of all the sub-sequences $\left(W_{n+1}^{i}, 1 \leq i \leq k_{n}\right)$. Remark that a pixel may appear several times in the sequence $W_{n+1}$.

\begin{tabular}{|l|l|l|}
\hline 4 & 3 & 2 \\
\hline 5 & $x$ & 1 \\
\hline 6 & 7 & 8 \\
\hline
\end{tabular}

FiguRE 2.3. Labeling of the neighbors of a point $x$

- If $W_{n}=\emptyset$, then $W_{n+1}=\emptyset$.

The subsequence $W_{n+1}^{i}$ may be seen as the offspring of the pixel $x_{i}$ and $\sharp W_{n+1}^{i}$ is distributed according to a binomial $\mathscr{B}(8, p)$ distribution. Therefore, the process $\left(\sharp W_{n+1}\right)_{n \geq 0}$ is distributed as a Galton-Watson process starting from a single individual and with offspring distribution $\mathscr{B}(8, p)$.

We now define a pruning procedure for the process $\left(W_{n}\right)_{n \geq 0}$ which defines a set-valued process $\left(\hat{W}_{n}\right)_{n \geq 0}$ as follows:

- $\hat{W}_{0}=\{(0,0)\}$.

- Suppose that we have constructed $\hat{W}_{0}, \hat{W}_{1}, \ldots, \hat{W}_{n}$ where each set $\hat{W}_{i}$ is composed of elements of $W_{i}$, with $\hat{W}_{n}=\left\{\hat{x}_{1}^{n}, \ldots, \hat{x}_{k}^{n}\right\}$ (ranked in increasing order). To every $\hat{x}_{i}^{n}$ there corresponds a point $x_{j_{i}}^{n}$ of $W_{n}$ and we set

$$
\hat{W}_{n+1}^{i}=W_{n+1}^{j_{i}} \backslash\left(\bigcup_{\ell=1}^{i-1} \bar{B}\left(\hat{x}_{\ell}^{n}\right) \bigcup_{r=0}^{n-1} \bigcup_{x \in \hat{W}_{r}} \bar{B}(x)\right)
$$

and then

$$
\hat{W}_{n+1}=\bigcup_{i=1}^{k} \hat{W}_{n+1}^{i} .
$$


- If $\hat{W}_{n}=\emptyset$, then $\hat{W}_{n+1}=\emptyset$.

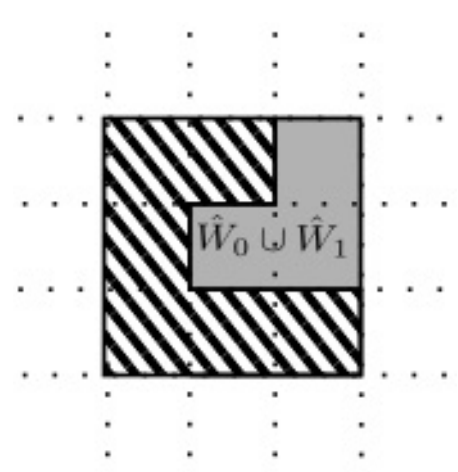

(a) $W_{1}=\{(0,1),(1,1)\}$

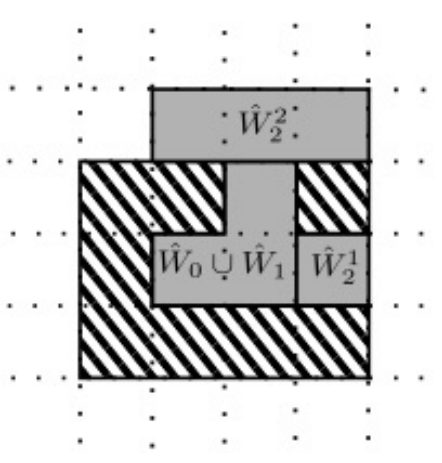

(b) $\quad W_{2}=W_{2}^{1} \cup W_{2}^{2}$ where

$W_{2}^{1}=\{(2,0),(1,1),(0,0),(0,-1)\}$ and

$W_{2}^{2}=\{(2,1),(2,2)(1,2),(0,2),(2,0)\}$

Figure 2.4. Example for the two first prunings

As the process $\left(\hat{W}_{n}\right)_{n \geq 0}$ is distributed as the process $\left(\tilde{W}_{n}\right)_{n \geq 0}$ (since the recursive formulas are the same), we have for every $a \geq 0$

$$
\mathbb{P}\left(\sharp \mathcal{C}_{0} \geq a\right)=\mathbb{P}\left(\sharp \bigcup \tilde{W}_{n} \geq a\right)=\mathbb{P}\left(\sharp \bigcup \hat{W}_{n} \geq a\right) .
$$

Furthermore, it is clear by construction (pruning procedure) that $\sharp \hat{W}_{n} \leq \sharp W_{n}$ for every $n \geq 0$, which yields

$$
\mathbb{P}\left(\sharp \bigcup \hat{W}_{n} \geq a\right) \leq \mathbb{P}\left(\sharp \bigcup W_{n} \geq a\right)=\mathbb{P}(\sharp Z \geq a)
$$

which ends the proof.

Remark 2.2. Looking at Figure 2.2, it seems that we could in fact take $m=5$ except for the first generation of the tree where we must keep $m=8$. The corresponding tree is not stricto sensu a Galton-Watson tree (as the offspring distribution is not always the same), however the computations of the next subsection can probably be adapted to this case. For sake of simplicity, we take $m=8$ in what follows to have a true Galton-Watson process.

2.4. Computation of the threshold. Let $\left(Z_{n}\right)_{n \geq 0}$ be a a sub-critical Galton-Watson process with offspring distribution a binomial $\mathscr{B}(m, p)$. For this offspring distribution to be sub-critical, recall that we must suppose that

$$
m p<1 .
$$

We need an upper bound for the probability $\mathbb{P}(\sharp Z \geq k)$. For this purpose, we use Dwass formula that relates the total population size of any sub-critical (or critical) Galton-Watson process to the associated Lukasiewicz random walk, see [11]:

Proposition 2.3. Let $\left(Z_{n}\right)_{n \geq 0}$ be a critical or sub-critical Galton-Watson process with offspring distribution $\nu$. Let $\left(Y_{n}\right)_{n \geq 1}$ be a sequence of i.i.d. random variables with distribution $\nu$. We set for every $n \geq 1$

$$
S_{n}=\sum_{i=1}^{n} Y_{i}
$$


Then we have

$$
\mathbb{P}(\sharp Z=n)=\frac{1}{n} \mathbb{P}\left(S_{n}=n-1\right) .
$$

Recall that $\nu$ is the binomial $\mathscr{B}(m, p)$ distribution; then for every $n \geq 1, S_{n}$ is distributed according to a binomial $\mathscr{B}(m n, p)$ distribution (as the sum of i.i.d $\mathscr{B}(m, p)$ random variables). Therefore

$$
\mathbb{P}(\sharp Z=n)=\frac{1}{n}\left(\begin{array}{c}
m n \\
n-1
\end{array}\right) p^{n-1}(1-p)^{m n-n+1} .
$$

This exact formula allows us to compute the looked after threshold:

Proposition 2.4. We set

$$
q=\frac{m^{m} p(1-p)^{m-1}}{(m-1)^{m-1}}, \text { and } C=\sqrt{\left(\frac{m}{m-1}\right)} \frac{e(1-p)}{p K^{2}} \frac{1}{1-q}
$$

with $K=e^{\frac{3}{2}\left(1-\ln \frac{3}{2}\right)}$. Then, for every $\varepsilon>0$, a threshold is given by

$$
a_{0}=\frac{\ln \frac{\varepsilon}{C}}{\ln q}
$$

that is for every $a \geq a_{0}$, we have

$$
\mathbb{P}(\sharp Z \geq a) \leq \varepsilon .
$$

Proof. By Proposition 2.3 and Equation (2.2), we get for every $a>0$

$$
\mathbb{P}(\sharp Z \geq a)=\sum_{n \geq a} \mathbb{P}(\sharp Z=n) \leq \sum_{n \geq a}\left(\begin{array}{c}
m n \\
n
\end{array}\right) p^{n-1}(1-p)^{m n-n+1},
$$

since $\frac{1}{n}\left(\begin{array}{c}m n \\ n-1\end{array}\right) \leq\left(\begin{array}{c}m n \\ n\end{array}\right)$.

We now use the following bounds for the factorial function whose proof is postponed to the end of this section:

Lemma 2.4. For every positive integer $n$, we have

$$
K n^{n+\frac{1}{2}} e^{-n} \leq n ! \leq n^{n+\frac{1}{2}} e^{-n} e
$$

with $K=\exp \left(\frac{3}{2}\left(1-\ln \frac{3}{2}\right)\right)$.

Using these bounds in the binomial coefficient, we get

$$
\begin{aligned}
\mathbb{P}(\sharp Z \geq a) & \leq \sum_{n \geq a} \frac{(m n)^{m n+\frac{1}{2}} e^{-m n} e}{K^{2} n^{n+\frac{1}{2}} e^{-n}(m n-n)^{m n-n+\frac{1}{2}} e^{-m n+n}} p^{n-1}(1-p)^{m n-n+1} \\
& \leq\left(\frac{m}{m-1}\right)^{\frac{1}{2}} \frac{e(1-p)}{p K^{2}} \sum_{n \geq a}\left(\frac{m^{m} p(1-p)^{m-1}}{(m-1)^{m-1}}\right)^{n} n^{-\frac{1}{2}} \\
& \leq\left(\frac{m}{m-1}\right)^{\frac{1}{2}} \frac{e(1-p)}{p K^{2}} \sum_{n \geq a} q^{n} .
\end{aligned}
$$


Let us remark that the function $x \longmapsto x(1-x)^{m-1}$ reaches its maximum on the interval $[0,1]$ at $x=\frac{1}{m}$. As we supposed that $p<\frac{1}{m}$, then

$$
q<\frac{m^{m} \frac{1}{m}\left(1-\frac{1}{m}\right)^{m-1}}{(m-1)^{m-1}}=1 .
$$

The previous sum is then finite and

$$
\mathbb{P}(\sharp Z \geq a) \leq\left(\frac{m}{m-1}\right)^{\frac{1}{2}} \frac{e(1-p)}{p K^{2}} \frac{q^{a}}{1-q}=C q^{a} .
$$

Therefore (recall that $q<1$ which gives $\ln q<0$ ),

$$
a \geq \frac{\ln \frac{\varepsilon}{C}}{\ln q} \Rightarrow C q^{a} \leq \varepsilon \Rightarrow \mathbb{P}(\sharp Z \geq a) \leq \varepsilon .
$$

Proof of Lemma 2.4. By the trapezoidal rule, as the logarithm function is concave, we have for every positive integer $k$

$$
\frac{\ln (k+1)+\ln k}{2} \leq \int_{k}^{k+1} \ln t \mathrm{~d} t
$$

This implies

$$
\begin{aligned}
\sum_{k=1}^{n-1} \frac{\ln (k+1)+\ln k}{2} \leq \int_{1}^{n} \ln t \mathrm{~d} t & \Leftrightarrow \ln n !-\frac{\ln n}{2} \leq n \ln n-n+1 \\
& \Leftrightarrow \ln n ! \leq\left(n+\frac{1}{2}\right) \ln n-n+1 \\
& \Leftrightarrow n ! \leq n^{n+\frac{1}{2}} e^{-n} e
\end{aligned}
$$

which gives the upper bound.

Still using the concavity of the logarithm function, its graph is always below its tangent which is is given at point $k$ by the equation

$$
y=\frac{1}{k} x-1+\ln k \text {. }
$$

This yields

$$
\begin{aligned}
\int_{k-1 / 2}^{k+1 / 2} \ln t \mathrm{~d} t & \leq \frac{\ln k+1 / 2 k+\ln k-1 / 2 k}{2}=\ln k \\
& \Rightarrow \int_{3 / 2}^{n+1 / 2} \ln t \mathrm{~d} t \leq \sum_{k=2}^{n} \ln k=\ln n ! \\
& \Rightarrow(n+1 / 2) \ln (n+1 / 2)-n-\frac{3}{2} \ln \frac{3}{2}+1 \leq \ln n ! \\
& \Rightarrow(n+1 / 2) \ln n+(n+1 / 2) \ln (1+1 / 2 n)-n-\frac{3}{2} \ln \frac{3}{2}+1 \leq \ln n !
\end{aligned}
$$

To get the lower bound, it suffices to check that

$$
(n+1 / 2) \ln (1+1 / 2 n) \geq 1 / 2
$$

which comes immediately from the inequality

$$
\forall x \geq 0, \ln (1+x) \geq x-\frac{x^{2}}{2} .
$$


2.5. Other cases. We gave all the details of the proofs with the 8-connectivity for $2 \mathrm{D}$ images but the arguments of the previous subsections can be adapted to many other situations. We present some of them without detailing the proof of the stochastic domination.

2.5.1. Other definitions of connectivity. We focused on the 8-connectivity but the same arguments work for the 4-connectivity (taking $m=4$ ). Numerical tests are performed in the next section to compare the performances of each connectivity.

We can also extend this work to epsilon-connectivity (see [19] for a more precise introduction) where two pixels are said to be connected if their $\ell^{\infty}$-distance is less than $k$. For instance, for $k=2$, this allows to fill small gaps between cracks that may be created because of the noise and/or the thickness of the cracks. All the previous computations remain valid taking $m=24$ since a neighborhood of a pixel is now a $5 \times 5$ square.

2.5.2. 3D images. We also may extend the results to 3D stacks. Recall that we consider a $3 \mathrm{D}$ stack whose size is $1287 \times 1287 \times 99$. If we consider the nearest neighbors of a pixel to define connectivity, the neighborhood of a pixel is now a cube of size 3 and the results apply with $m=26$. Some numerical experiments are presented in Section 3.4.

2.5.3. Diameter of the connected component. Let us go back to 2D-images and 8-connectivity. Let $\mathcal{C}_{0}$ be the connected component of the image that contains 0 . For $x, y \in \mathcal{C}_{0}$, we set $\Gamma_{x, y}$ as the set of connected paths that go from $x$ to $y$ remaining inside $\mathcal{C}_{0}$. For $\gamma \in \Gamma_{x, y}$, we denote by $\ell(\gamma)$ its length i.e. the number of pixels $\gamma$ is composed with. Finally, we define the diameter of $\mathcal{C}_{0}$ by

$$
\operatorname{diam}\left(\mathcal{C}_{0}\right)=\max _{x, y \in \mathcal{C}_{0}} \min _{\gamma \in \Gamma_{x, y}} \ell(\gamma)
$$

This diameter can be used to discriminate cracks from noise. It also discriminates cracks (that have a quite large diameter) from ball-like connected components with the same number of pixels. Moreover our method still apply since we have, for every positive integer $a$,

$$
\mathbb{P}\left(\operatorname{diam}\left(\mathcal{C}_{0}\right) \geq 2 a\right) \leq \mathbb{P}\left(Z_{a}>0\right)
$$

where $\left(Z_{n}\right)_{n \geq 0}$ is a Galton-Watson process with binomial $\mathscr{B}(m, p)$ offspring distribution with $m=8$. Indeed, if $\operatorname{diam}\left(\mathcal{C}_{0}\right) \geq 2 a$, there exists two points $x, y \in \mathcal{C}_{0}$ whose shortest path between them has length greater than $2 a$. Then, at least one of the shortest paths from the origin to $x$ or $y$ is greater than $a$ and the Galton-Watson process needs at least $a$ generations to attain this point.

To compute the looked after threshold, we use the following lemma.

Lemma 2.5. Let $\left(Z_{n}\right)_{n \geq 0}$ be a Galton-Watson process with binomial $\mathscr{B}(m, p)$ offspring distribution. Then, for every positive integer $n$, we have

$$
\mathbb{P}\left(Z_{n}>0\right) \leq(m p)^{n} .
$$

Proof. Let us denote by $F(z)$ the generating function of the binomial $\mathscr{B}(m, p)$ distribution:

$$
F(z)=(p z+1-p)^{m} .
$$

Let us also denote for every $n \geq 0$ by $F_{n}(z)$ the generating function of $Z_{n}$. Then we have the classical recursive formula:

$$
\forall n \geq 0, \quad F_{n+1}(z)=F\left(F_{n}(z)\right)=F_{n}(F(z)) .
$$


Moreover, we have $\mathbb{P}\left(Z_{n}>0\right)=1-F_{n}(0)$ and $\mathbb{P}\left(Z_{0}>0\right)=1$. Therefore, we get

$$
\begin{aligned}
\mathbb{P}\left(Z_{n+1}>0\right) & =1-F_{n+1}(0) \\
& =1-\left(1-p\left(1-F_{n}(0)\right)^{m}\right. \\
& \leq m p\left(1-F_{n}(0)\right)=m p \mathbb{P}\left(Z_{n}>0\right) .
\end{aligned}
$$

The result follows from an easy induction.

In that case, the threshold satisfies

$$
a_{0}=2 \frac{\log \varepsilon}{\log (m p)} .
$$

This second threshold gives a geometric information, since we only keep structures with large diameters. In our case, this favors the cracks, that have anisotropic structure, with respect to the noise that can be viewed as small isotropic elements. Numerical tests, are reported in subsection 3.1.4.

\section{Application to CRACK Detection}

As already mentioned, the application we have in mind is the identification of the cracks that appear in granite samples. Here, we are interested in denoising as a post-processing final step in for a complete crack identification method (based on a variational formulation) that we briefly describe in the sequel. That is why we focus on binary images (though the method works with grey level images as well) and the impulse noise that has to be deleted. The grain filter is a very efficient tool to achieve this goal: that is why we decided to focus on some improvements and automatic thresholding.

The numerical experimentation ${ }^{2}$ has been performed with MATLAB. We do not report on the computation time (we used different machines, and the codes are not optimized, especially in the 3D case). However, we may emphasize that the most time consuming step is the computation of the size (or geodesic diameter) of connected components. We used a built in MATLAB function, but this step should/can be improved with ad-hoc methods.

3.1. Tests with a 2D synthetic black and white image. We first perform numerical tests on a synthetic image that has been built with the image of Figure 1.1(a). It has been manually modified to get a image close to real data. This will be considered as the ground truth and we add impulse noise whose level is $p$, for $p \in[0.01,0.35]$. The image is presented in Figure 3.1. We assume that $m$ (the number of neighbors in the chosen neighborhood) and $\varepsilon$ (the test level) are given. A typical choice for $m$ is $m=3^{2}-1=8$ for $2 \mathrm{D}$ images (though we shall test $m=4$ as well) and $m=3^{9}-1=26$ for $3 \mathrm{D}$ images (using $3 \times 3 \times 3$ neighborhoods). The parameter $\varepsilon$ is chosen small enough. We will make this precise in the sequel, when we study the sensitivity with respect to $\varepsilon$.

Let us mention that we should only consider $p<0.125$ since we have chosen $m=8$ and we have to fit the assumption (2.1). However, we have added noise up to level 0.35. In the case where $p$ is known (it is the noise level) the algorithm writes

\footnotetext{
${ }^{2}$ All the images of this section, can be download here http://maitinebergounioux.net/PagePro/ Publications_files/Images_JMIV.rar
} 




(a) Ground truth (synthetic image)

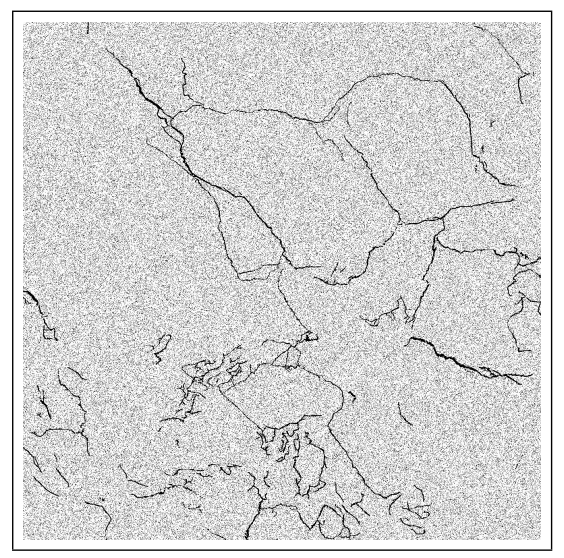

(b) Impulse noise with $\mathrm{p}=0.15$

FiguRE 3.1. Ground truth and noisy image

However, in the real data case, the probability $p$ is unknown and its estimate is an important issue (that also determines the $m$ value). We may first estimate it using the number of black pixels of the binary image divided by its total size $M: p \simeq \frac{\operatorname{Size}(u=1)}{M}$. This rough method gives an upper bound for $p$ that is too large an estimate. Indeed, the number of black pixels includes both the noise and the cracks, whereas we only want to count the noisy pixels. So we adopt a multi-scale like strategy to perform successive updates of $p$ once we have performed a more accurate selection of cracks pixels.

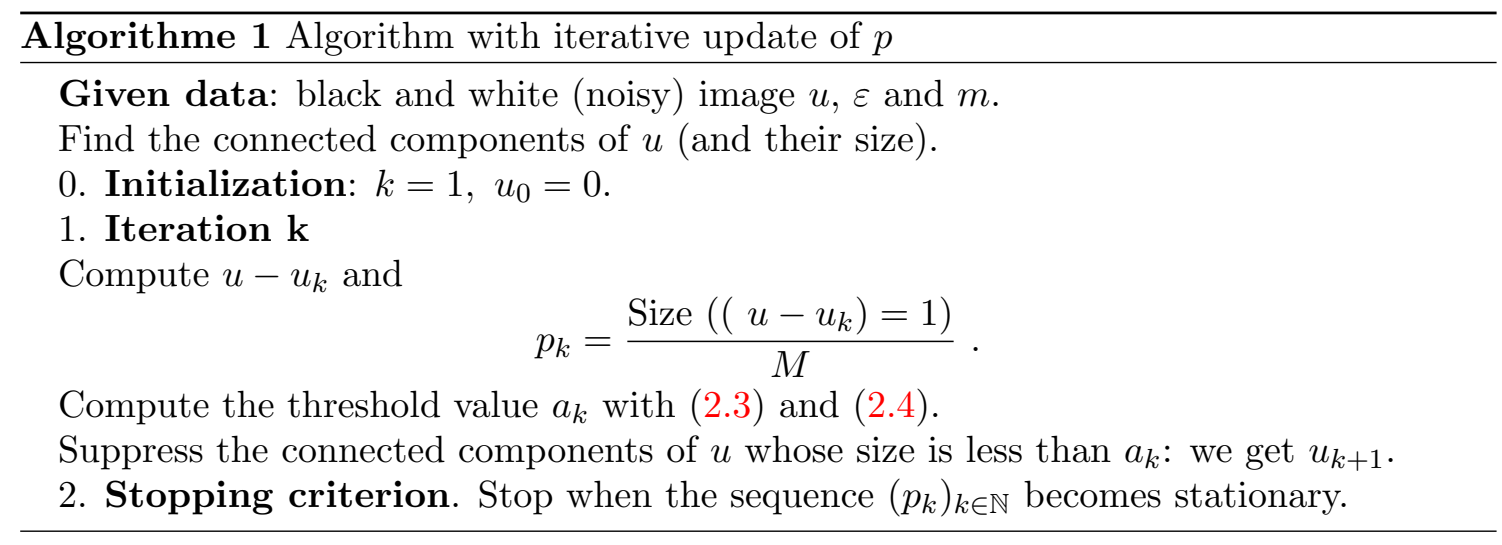

We report in Table 3.1 the values of the computed probability $p$ and the threshold with and without this strategy with respect to the noise level. This corresponds to the computed values at iteration 1 of Algorithm (1) and at the last iteration Algorithm (1) . We have set the maximal number of iterations at 20 . 


\begin{tabular}{|c|c|c|c|c|c|}
\hline $\begin{array}{c}\text { Noise } \\
\text { level }\end{array}$ & $\begin{array}{l}\text { Computed } \\
\text { probability } \\
\text { Iteration } 1\end{array}$ & $\begin{array}{c}\text { Computed } \\
\text { probability } \\
\text { Last iteration }\end{array}$ & $\begin{array}{l}\text { Threshold } \\
\text { (\# pixels) } \\
\text { Iteration } 1\end{array}$ & $\begin{array}{l}\text { Threshold } \\
\text { (\# pixels) } \\
\text { Last iteration }\end{array}$ & $\begin{array}{c}\text { Number } \\
\text { of } \\
\text { iterations } \\
\text { in Algorithm (1) }\end{array}$ \\
\hline 0.01 & 0.032 & 0.0096 & 26 & 11 & 3 \\
\hline 0.02 & 0.042 & 0.019 & 38 & 16 & 3 \\
\hline 0.03 & 0.051 & 0.029 & 54 & 23 & 3 \\
\hline 0.04 & 0.062 & 0.038 & 79 & 33 & 3 \\
\hline 0.05 & 0.071 & 0.048 & 121 & 48 & 4 \\
\hline 0.06 & 0.081 & 0.058 & 201 & 71 & 4 \\
\hline 0.07 & 0.091 & 0.068 & 354 & 107 & 4 \\
\hline 0.08 & 0.1 & 0.080 & 766 & 191 & 4 \\
\hline 0.09 & 0.11 & 0.093 & 2266 & 435 & 5 \\
\hline 0.10 & 0.12 & 0.12 & 26284 & 26284 & 2 \\
\hline 0.11 & 0.13 & 0.130 & 2347 & 2347 & 2 \\
\hline 0.12 & 0.14 & 0.131 & 2648 & 1633 & 20 (Max) \\
\hline 0.125 & 0.14 & 0.131 & 1521 & 1584 & 20 (Max) \\
\hline
\end{tabular}

TABLE 3.1. Computed probability and threshold with respect to the noise level, with Algorithm (1) with (last iteration) and without (first iteration) the adaptative strategy- $m=8, \varepsilon=10^{-6}$.

We can also see in Figure 3.2 the behavior of the computed threshold with respect to the noise level for the different approaches. We clearly see the change of behavior for a noise level greater than 0.11. This is consistent with the theoretical assumption (2.1).

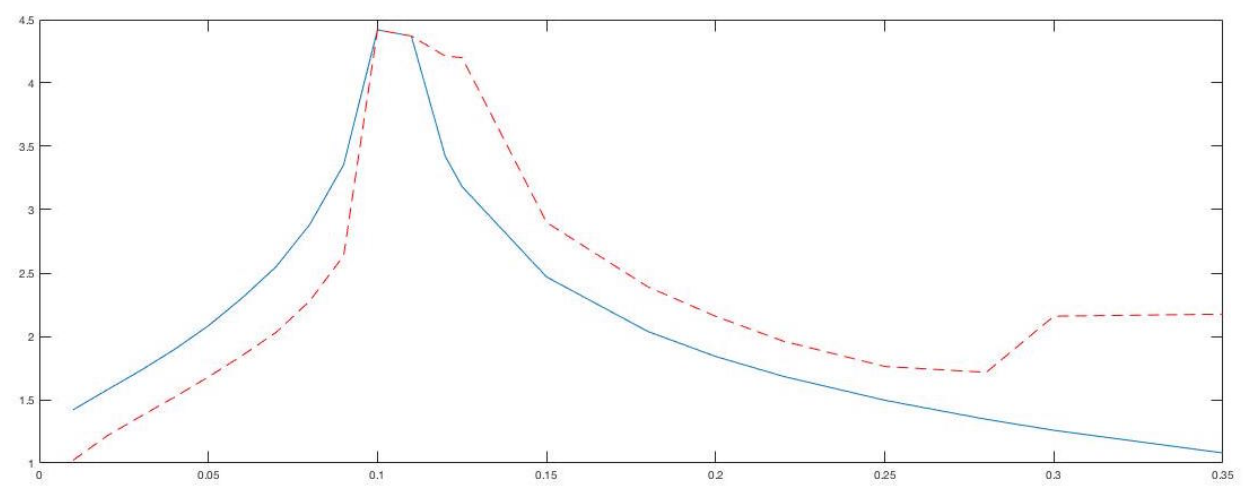

FiguRE 3.2. Evolution of the threshold with respect to the noise level in the range $\left[\begin{array}{ll}0 & 0.35\end{array}\right]$ - $\mathrm{X}$ axis is the noise level $p$ and $\mathrm{Y}$-axis is the threshold value (number of pixels) with $\log 10$ - scale. The continuous line corresponds to the case where $p$ is computed once (iteration 1) and the dotted curve to the computed threshold with adaptative strategy (last iteration of Algorithm (1)) $-m=8, \varepsilon=10^{-6}$. 


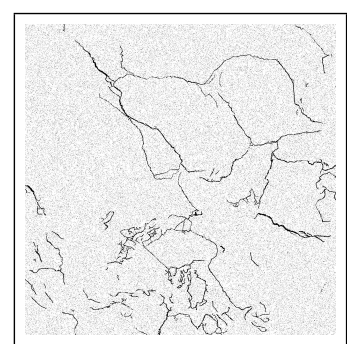

(a) Level noise $=0.05$

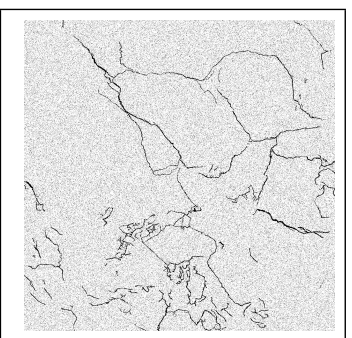

(e) Level noise $=0.09$

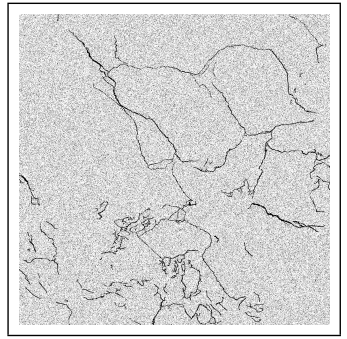

(i) Level noise $=0.15$



(b) Computed Solution

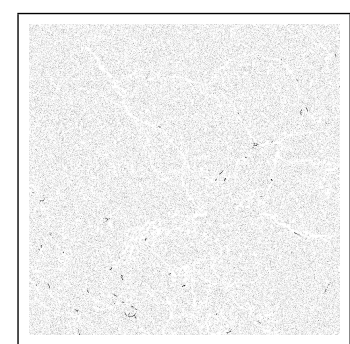

(c) Difference with the data

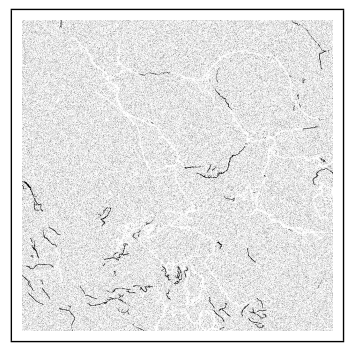

(g) Difference with the data

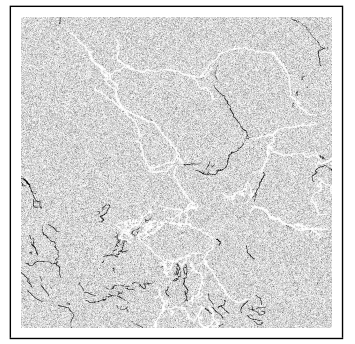

(k) Difference with the data

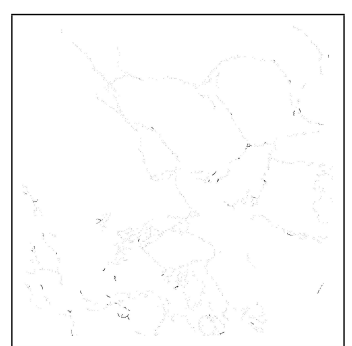

(d) Difference with the ground truth

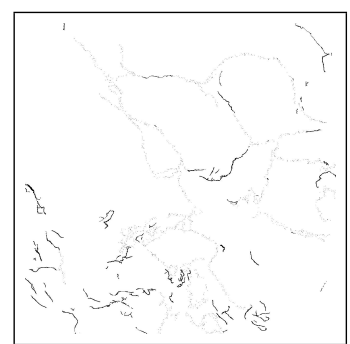

(h) Difference with the ground truth

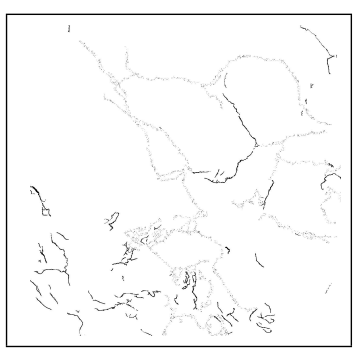

(j) Computed Solution



(1) Difference with the ground truth

FiguRE 3.3. Noisy image, solution given by Algorithm (1) and difference with the ground truth, for different noise levels - $m=8, \varepsilon=10^{-6}$ - The differences are presented as negative images for a better insight (white is equal to 0 , black is maximum).

3.1.1. Sensibility with respect to $\varepsilon$. We now investigate the sensibility of the solution computation with respect to $\varepsilon$. A typical choice of $\varepsilon$ is the inverse of the number of connected components as, by independence, the mean number of false positives would be 1 in this case. 


\begin{tabular}{|c|c|c|c|c|c|c|}
\hline Noise & \multicolumn{3}{|c|}{ Probability } & \multicolumn{3}{|c|}{ Threshold (\# pixels) } \\
\hline level & $\varepsilon=10^{-2}$ & $\varepsilon=10^{-4}$ & $\varepsilon=10^{-6}$ & $\varepsilon=10^{-2}$ & $\varepsilon=10^{-4}$ & $\varepsilon=10^{-6}$ \\
\hline 0.01 & 0.0095 & 0.0095 & 0.0096 & 5 & 8 & 11 \\
\hline 0.03 & 0.0287 & 0.0288 & 0.0290 & 11 & 17 & 24 \\
\hline 0.05 & 0.0477 & 0.0480 & 0.0483 & 22 & 35 & 48 \\
\hline 0.10 & 0.1203 & 0.1203 & 0.1203 & 158 & 215 & 271 \\
\hline 0.15 & 0.1496 & 0.1509 & 0.1517 & 476 & 635 & 792 \\
\hline
\end{tabular}

TABLE 3.2. Computed probability and threshold with respect to the noise level, with the adaptative strategy Algorithm (1) and different values of $\varepsilon$ $-\mathrm{m}=8$.



(a) $\varepsilon=10^{-2}$.



(b) $\varepsilon=10^{-4}$.



(c) $\varepsilon=10^{-6}$.

Figure 3.4. Examples of solutions given by Algorithm (1) for $\varepsilon=$ $10^{-2}, 10^{-4}, 10^{-6}$ - Noise $=0.05$.

This comparison shows that the role of $\varepsilon$ has not a big influence when the noise level belongs to $[0,0.125]$ (the image size is $1.65310^{6}$ ). In the sequel, we choose $\varepsilon \simeq 1 / M$ where $M$ is the size of $u$ that is, roughly speaking, $\varepsilon=10^{-6}$.

3.1.2. Sensibility with respect to the connectivity. We compare here the results obtained with these two different connectivities corresponding to $m=4$ and $m=8$. As expected, the behavior of the threshold value for $m=4$ changes for a noise level equal to 0.25 , which is consistent with Assumption (2.1).

Next figure presents the solutions that are obtained with Algorithm (1) for the same noisy data and $\mathrm{m}=4,8$ respectively. 


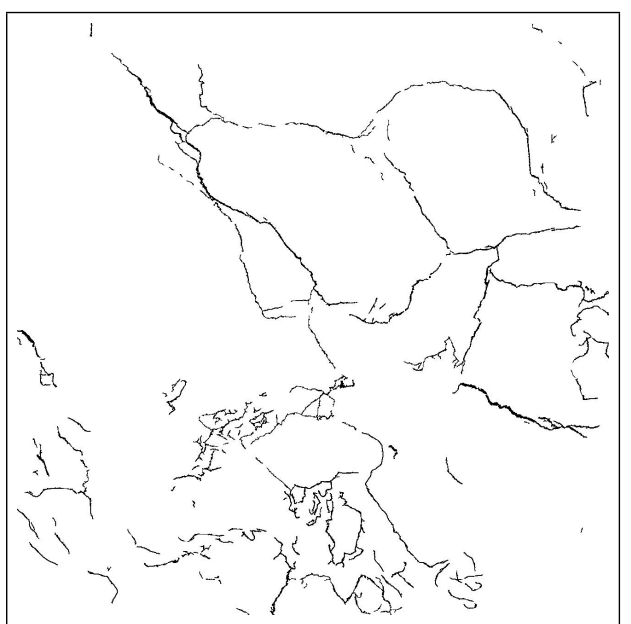

(a) Noise: $0.01, \mathrm{~m}=4$.

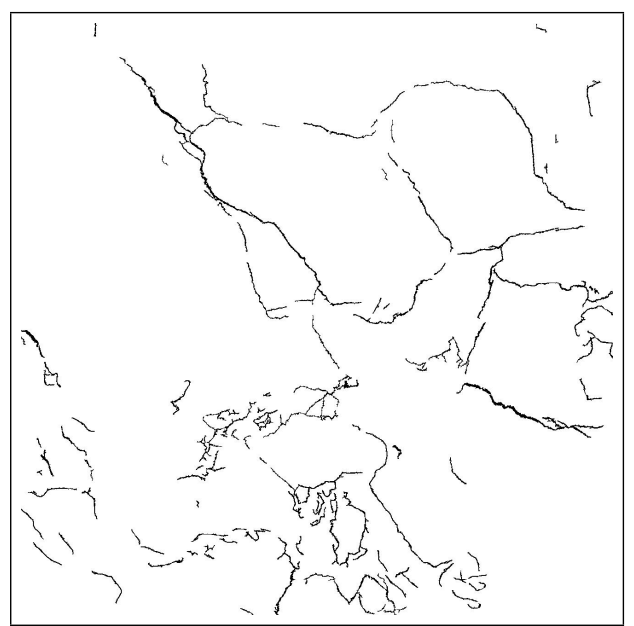

(c) Noise: $0.05, \mathrm{~m}=4$.



(e) Noise: $0.15 \mathrm{~m}=4$.

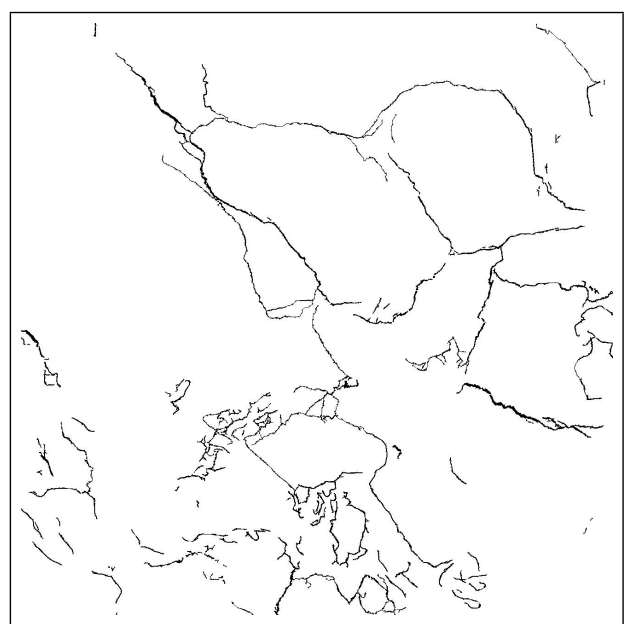

(b) Noise: $0.01, \mathrm{~m}=8$.

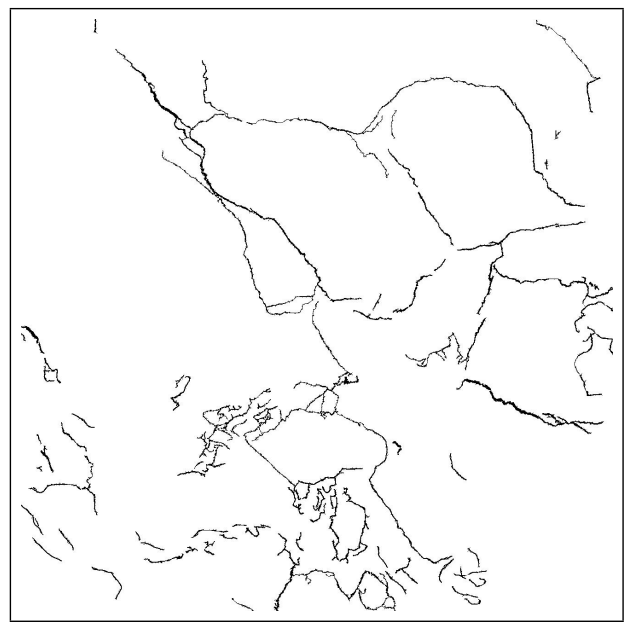

(d) Noise: 0.05, $\mathrm{m}=8$.

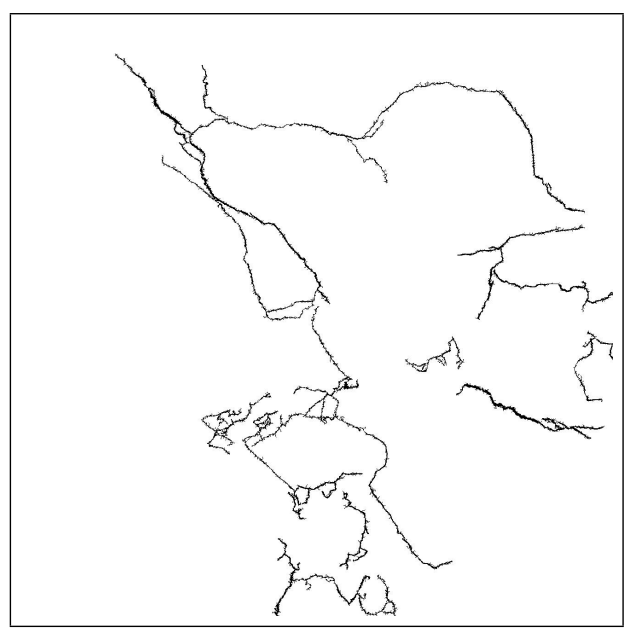

(f) Noise: $0.15, \mathrm{~m}=8$.

Figure 3.5. Comparison of the solutions computed with Algorithm (1) for $m=4, m=8$ and $\varepsilon=10^{-6}$. 
We can see on Figure 3.5 that the use of 4-connectivity gives worse results. Indeed, the use of a 4-neighborhood does not take the diagonal connections into account. Therefore, thin cracks are lost and thick ones may be disconnected.

3.1.3. Comparison with other filters and/or other thresholds. Though, there are a lot of filters to deal with impulse noise we limit our comparison to the median one and a morphological one, namely an opening filter with two different structural elements: a disk of radius 1 (which corresponds to the 4 -connectivity) and a square $3 \times 3$ (which corresponds to the 8-connectivity). Results are reported in Figure 3.6 for a noise level of 0.05.

Visually speaking the solution that we get with Algorithm (1) is the best one. If we compare with the ground truth, we get a quantitative estimate of the performance, with $\delta_{1}$ which is the $\ell^{1}$ norm of the difference between the ground truth and the solution.



(a) Median filter $3 \times 3, \delta_{1}=6371$.



(b) Opening with disk of radius , $\delta_{1}=$ 9900 .

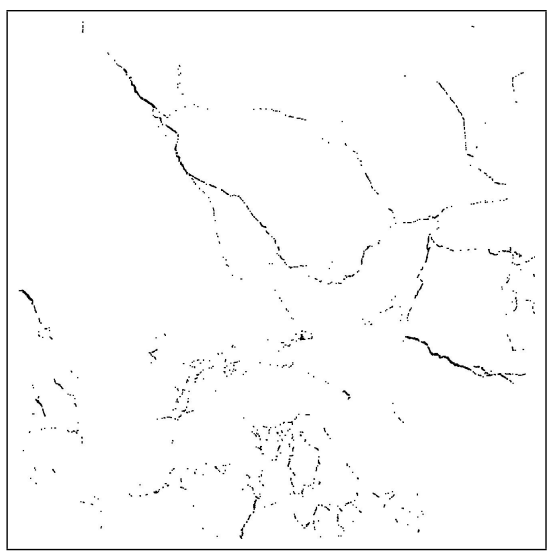

(c) Opening with square of size $3 \times 3$, $\delta_{1}=21344$.



(d) Algorithm (1), $\mathrm{m}=8, \varepsilon=10^{-6}$, $\delta_{1}=3621$.

Figure 3.6. Comparison of the solutions computed with different filters, for a noise level equal to 0.05 . Here $\delta_{1}$ is the $\ell^{1}$ norm of the difference between the ground truth and the solution, that is the number of pixels that are different. 
3.1.4. Use of the connected components diameter. As mentioned in subsection 2.5.3, we can use Algorithm (1) and consider the geodesic diameter of connected components instead of the size. The modified algorithm writes

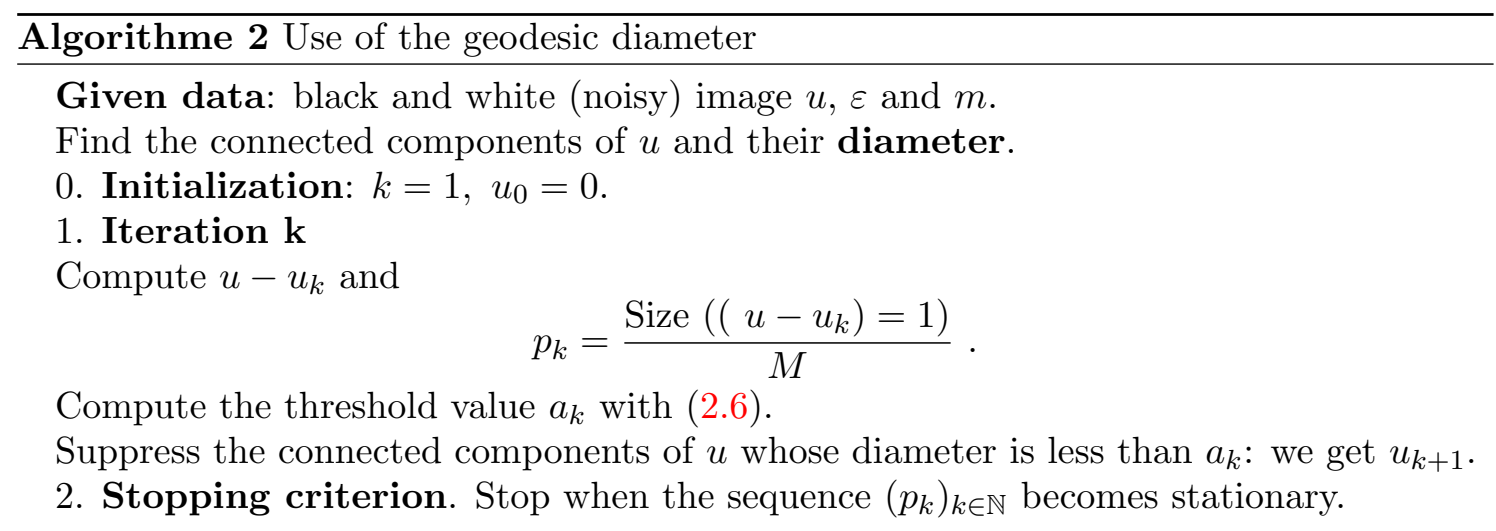



(a) Ground truth.

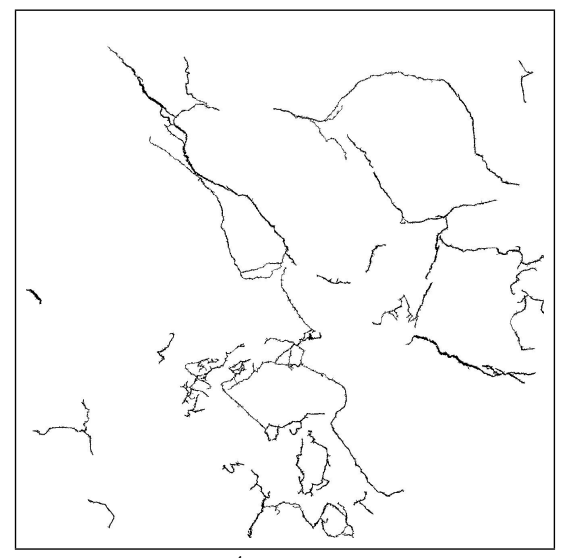

(c) $\varepsilon=10^{-4}$ (threshold $\left.=51\right)$.

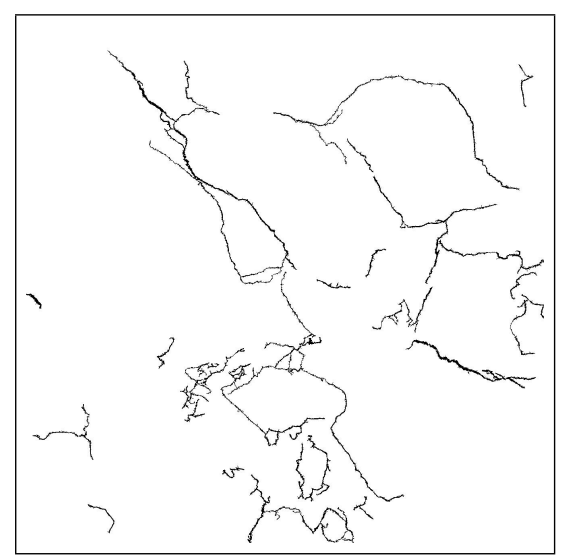

(b) $\varepsilon=10^{-3}$ (threshold $=37$ ).

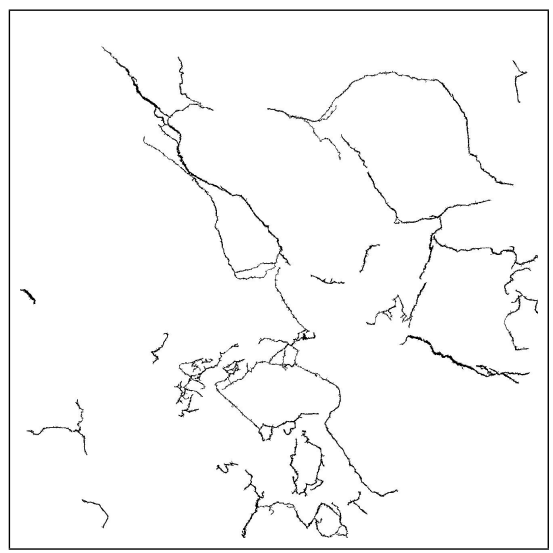

(d) $\varepsilon=10^{-6}$ (threshold $=87$ ).

Figure 3.7. Solutions computed with Algorithm (2) for $m=8$ and different values of $\varepsilon$. 
The use of the geodesic diameter is not appropriate if the noise is too large. Indeed, if $m p$ is too close to 1 then the threshold $a_{0}=2 \frac{\log \varepsilon}{\log m p}$ becomes quite large and we loose most of the crack information. If the noise is small enough, then we get good results provided $\varepsilon$ is not too large. However, the method seems to be robust with respect to small $\varepsilon$. Figures 3.7 and 3.8 present the results for a noise level equal to 0.09 and $m=8$



(a) Solution with Algorithm (1).

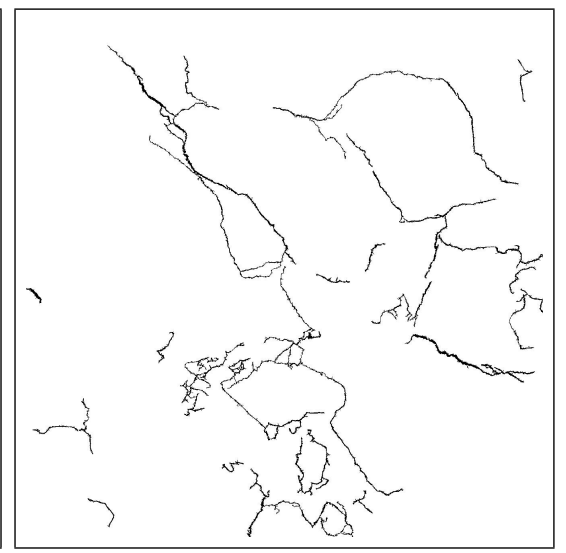

(b) Solution with Algorithm (2).



(c) Difference with the ground truth.

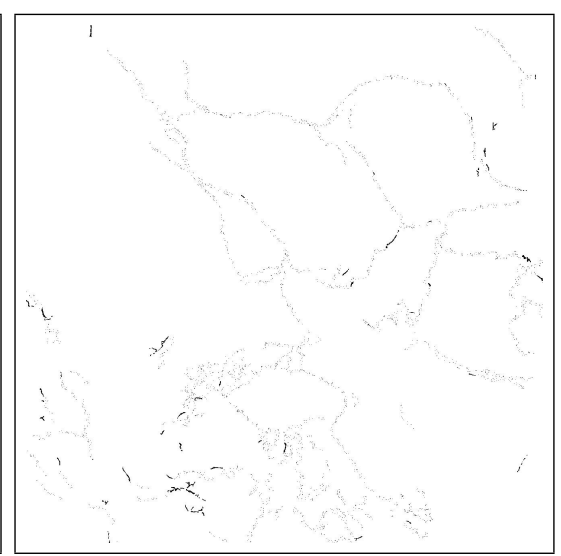

(d) Difference with the ground truth.

Figure 3.8. Comparison of the solutions computed with Algorithms (1) and (2) for $m=8, \varepsilon=10^{-4}$ and a noise level 0.09 .

\subsection{Tests with $2 \mathrm{D}$ a real black and white image.}

3.2.1. Preprocessing. We present some results on a 2D slice of a granite sample stack, for the sake of simplicity (see Figure 1.1 (a)). Though, it is not our purpose here, we briefly present the preprocessing method:

(1) Original (2D or 3D) images are pre-processed with the method of $[3,4]$. More precisely, we use a variational model that allows to split the image in three components denoted $v, u$ and $w$ :

- the $v$ component is a smooth (continuous) function whose distributional derivative is of bounded variation: it represents the image dynamic; 
- the $u$ component is a bounded variation function and ideally, should be piecewise constant. It is called a cartoon component: the image contours are given by the jump set of $u$;

- the $w$ component is a $L^{2}$ function that represents the details and/or the noise. The main inconvenient of variational methods is their dependence to some parameters. The result of the above decomposition depends on the choice of such parameters. We refer to [4] to get tuning rules for the two parameters $\lambda$ and $\mu$ to be used in this context. From a practical point of view we choose $\mu \simeq 1.4 \lambda$. The tests we present here use $\lambda=5, \mu=7$.

The preprocessing method is robust enough with respect to the choice of parameters and we can get rid of most noise (involved in the $w$ component). So we focus on the cartoon component $u$ that involves all the contours. However, though the gaussian part of the noise has been removed, impulse noise still remains. The challenge is to distinguish the cracks that are thin structures from the noise (which is unstructured). However, it will be still quite difficult to recover micro-cracks.

(2) Next, the $u$ component has to be binarized. This is a delicate step since the threshold parameter is not easy to find. We decided to choose $s=m(u)-\sigma(u)$ where $m$ and $\sigma$ are the mean value and the standard deviation respectively of $u$. This is an heuristic choice that we cannot fully justify by now.

(3) Last, we use the thresholded (binary) image and use Algorithm (1).



(a) Original (2D) image.



(c) $v$ component.



(b) $u$ component.

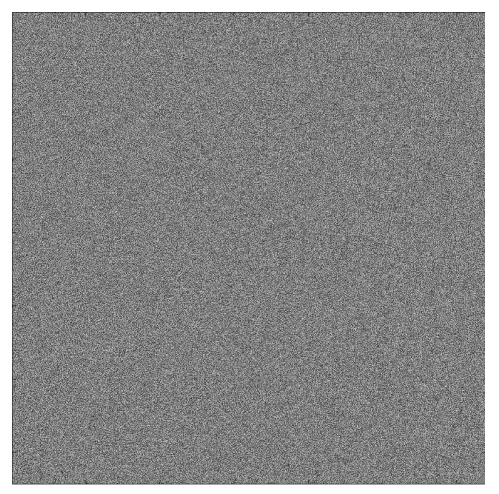

(d) $w$ component.

FiguRE 3.9. Image decomposition with $\lambda=5$ and $\mu=7$ - The contrast and lightning have been emphasized to provide a better view of the images. 


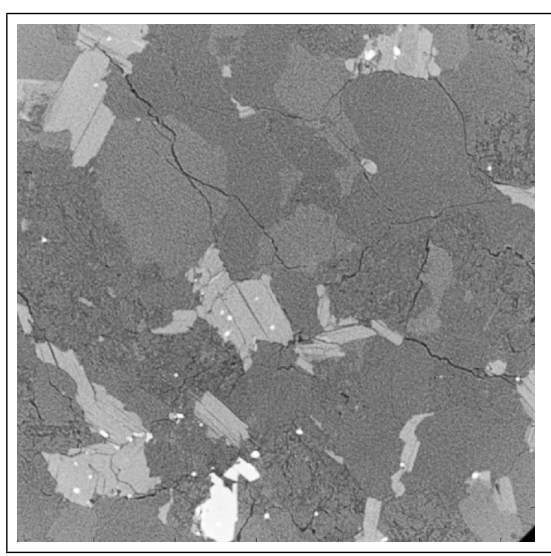

(a) $u$ component.

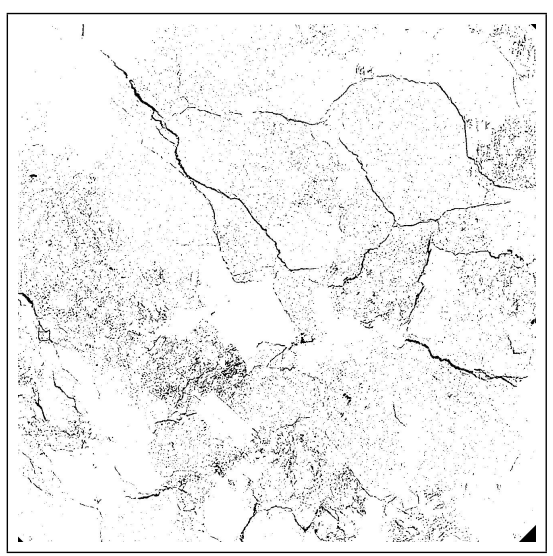

(b) Thresholded $u$ component.

Figure 3.10. Thresholded component used as the binary data

3.2.2. $2 D$ results. From now, we use the binary thresholded cartoon component as the data (see Figure 3.10). We report in Table 3.3 the evolution of $p$ estimate. We see that the iterative process becomes stationary very quickly ( 5 iterations).

\begin{tabular}{|c||c|c|c|c|}
\hline Iteration & 1 & 2 & 3 & 4 \\
\hline$p_{k}$ & $4.692810^{-2}$ & $3.365610^{-2}$ & $3.014610^{-2}$ & $2.917110^{-2}$ \\
\hline$a_{k}$ & $7.482710^{7}$ & $4.618310^{7}$ & $4.065910^{7}$ & $3.923610^{7}$ \\
\hline
\end{tabular}

TABLE 3.3. Computed probability $p_{k}$ and corresponding threshold $a_{k}$ during the iterations $-m=8$ and $\varepsilon=10^{-6}$.

Next figure shows the solution with Algorithms (1) and (2) :



(a) Noisy data.

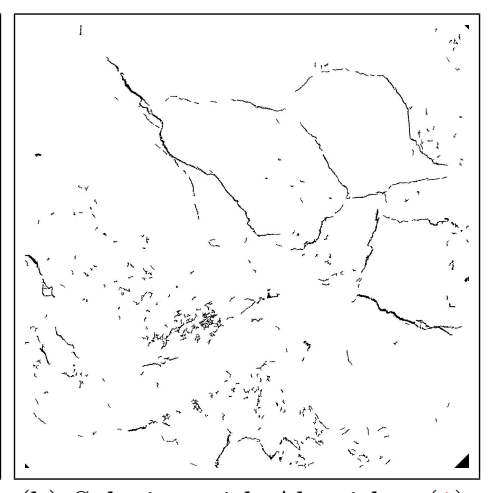

(b) Solution with Algorithm (1).

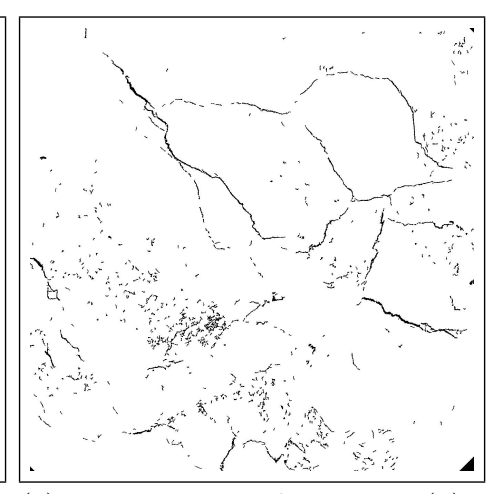

(c) Solution with Algorithm (2).

Figure 3.11. Solutions computed by Algorithms (1) and (2) with $m=8$ and $\varepsilon=10^{-6}$. 
3.3. Grey level images. We have considered binary images, which implies some thresholding process when dealing with gray-level images. However, following [8] we may adapt the algorithm by considering the image level sets. Precisely, for each level $k \in[0,255]$, we consider the thresholded image $u_{k}=\mathbf{1}_{u \geq k}$ (where $\mathbf{1}_{A}$ is the indicator function of the set $A$ ) and we recover the grey level solution as $u^{*}=\sum_{k=0}^{255} u_{k}$. This gives the following algorithm



Next figure shows the solution with the image of Lena, for $m=4$ and 8 respectively.



(a) Noisy data - noise level 0.05 .



(d) Noisy data - noise level 0.15 .



(b) Solution, $\mathrm{m}=4$.



(e) Solution, $\mathrm{m}=4$.

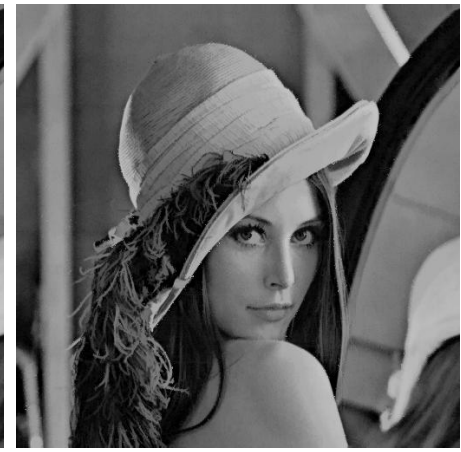

(c) Solution, $\mathrm{m}=8$.



(f) Solution, $\mathrm{m}=8$.

Figure 3.12. Lena denoising for two different noise levels $-\varepsilon=10^{-6}$ and $m=4,8$. 
We end this subsection with Algorithm (3) applied to the cartoon image of the granite image (before performing thresholding).



(a) $u$ component.

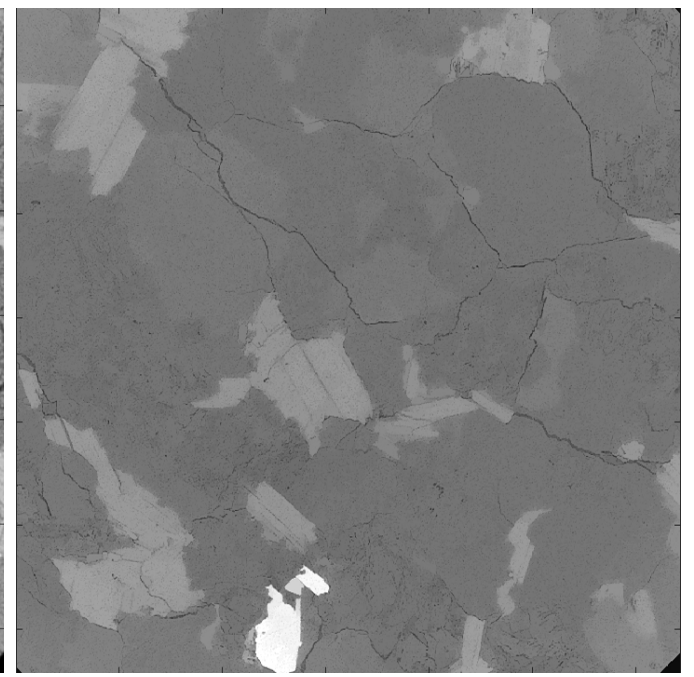

(b) Solution.

Figure 3.13. Granite denoising Cartoon part $-\varepsilon=10^{-6}$ and $m=8$.

We could now use an a posteriori thresholding process on this solution as well to recover the cracks, but this will not be very good since one can see that we have lost the microcracks structure.

3.4. 3D images. Though, we have presented the method with $2 \mathrm{D}$ images (whose domains are $\llbracket-N, N \rrbracket^{2}$ ) we may extend the result to 3D stacks. Recall that we consider a 3D stack whose size is $1287 \times 1287 \times 99$. If we consider the nearest neighbors of a pixel to define connectivity, the neighborhood of a pixel is now a cube of size 3 and the results apply with $m=26$ and Algorithm (1) works similarly in the 3D case. The main difference was the choice of the built in MATLAB function to compute the connected components directly with the 3D stack (and $m=26$ ).

The preprocessing was performed directly on the 3D stack (and not slice by slice) as explained in [4] and it was natural to use the 3D stack to perform binarization and denoising with Algorithm (1). Of course, the computation time is quite large since we have to estimate the size of a great number of connected components. Therefore, we have to develop further strategies to improve the $3 \mathrm{D}$ method as for example parallelization. 


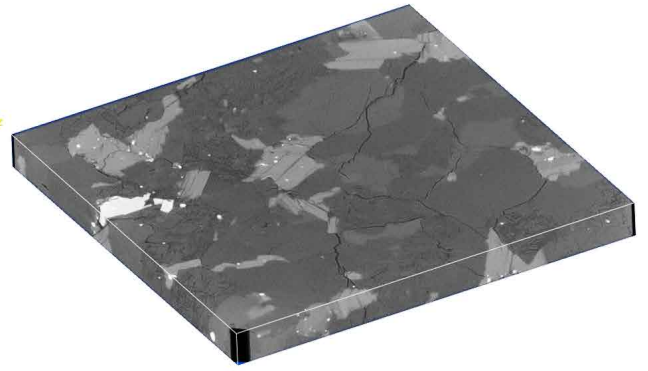

(a) Original Image.



(c) Noise component $w$.



(b) Smooth component $v$.



(d) Cartoon component $u$.

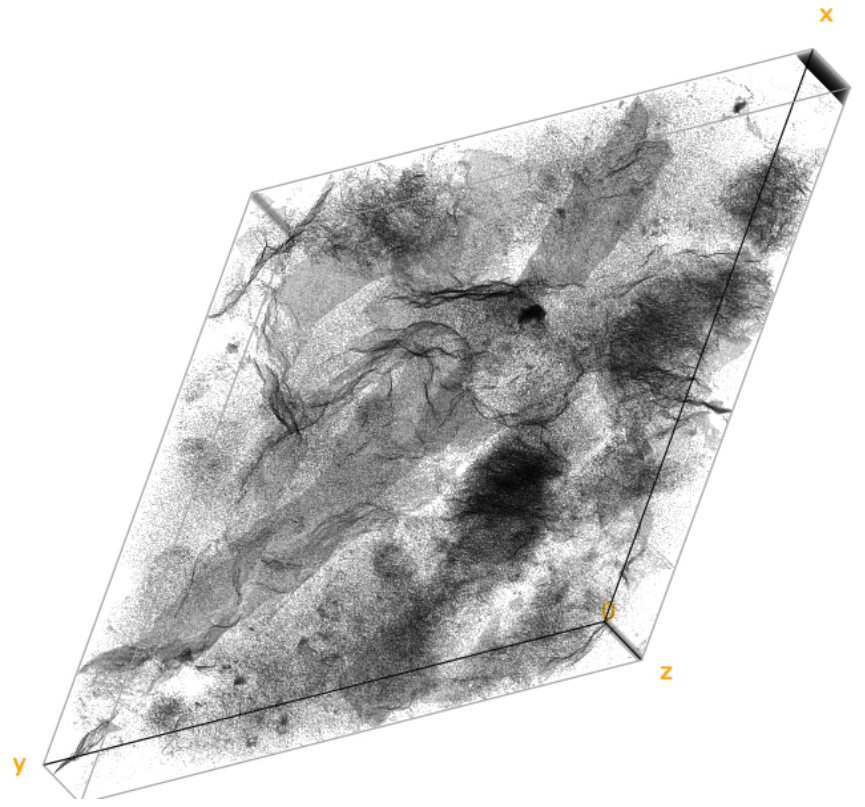

(e) Binarized cartoon component.

FiguRE 3.14. Decomposition of the 3D stack and binarized cartoon component.

Next figure shows the solution computed with $\operatorname{Algorithm(1),~} m=26$ and $\varepsilon=10^{-6}$. 




Figure 3.15. Solution.

\section{Conclusion}

The automatic threshold we propose seems to be performing and we are able to recover a lot of information from the binary images. Though, we have presented the method on a 2D material image, it can be applied to any binary images arising from appropriate pre-processings. One may think of as angiography images for example.

However, we could say that the size of the connected components is rather a crude criterion for distinguishing cracks for noise and some other geometric characteristic (as the diameter) would better discriminate our features. The key point is that we need an upper bound for the tail probability of the looked after characteristic (we obtained such a bound for $\mathbb{P}(\sharp Z \geq k)$ in the case of the total population size, and for $\mathbb{P}\left(Z_{n}>0\right)$ in the case of the diameter) which is not obvious in general (see for instance [1] for recent works in this direction).

Another point that we did not really focus on is the estimation of the parameter $p$ as it was not the central point of this work. Our method (especially after iterations) seems rather satisfactory but always overestimates this probability. Some more sophisticated methods have been developed in the literature and may be used to improve the results.

\section{REFERENCES}

[1] Louigi Addario-Berry, Luc Devroye, and Svante Janson. Sub-gaussian tail bounds for the width and height of conditioned Galton-Watson trees. Ann. Probab., 41(2):1072-1087, 032013.

[2] Krishna B. Athreya and Peter E. Ney. Branching processes. Dover Publications, Inc., Mineola, NY, 2004. Reprint of the 1972 original [Springer, New York; MR0373040].

[3] Maïtine Bergounioux. Mathematical Analysis of a Inf-Convolution Model for Image Processing. J. Optim. Theory Appl., 168(1):1-21, 2016.

[4] Maïtine Bergounioux. Second order decomposition model for image processing : numerical experimentation. In G. Bergounioux, M .and Peyré, C. Schnörr, J.-B. Caillau, and T. Haberkorn, editors, Variational Methods In Imaging and Geometric Control, volume 18 of Radon Series on Computational and Applied Mathematics, pages 5-34. Degruyter, 2017.

[5] Vicent Caselles and Pascal Monasse. Grain filters. J. Math. Imaging Vision, 17(3):249-270, 2002.

[6] Sylvie Chambon. Detection of points of interest for geodesic contours: application on road images for crack detection. In International Joint Conference on Computer Vision Theory and Applications, VISAPP, page sp, 2011. 
[7] Nicolas Coudray, Argyro Karathanou, and Sylvie Chambon. Multi-resolution approach for fine structure extraction: Application and validation on road images. In International Joint Conference on Computer Vision Theory and Applications, VISAPP, page sp, 2010.

[8] David Coupier, Agnès Desolneux, and Bernard Ycart. Image denoising by statistical area thresholding. J. Math. Imaging Vision, 22:183-197, 2005.

[9] A. Desolneux, L. Moisan, and J.-M. Morel. Meaningful alignments. 40(1):7-23, 2000.

[10] Agnes Desolneux, Lionel Moisan, and Jean-Michel Morel. From gestalt theory to image analysis: a probabilistic approach, volume 34. Springer Science \& Business Media, 2007.

[11] Meyer Dwass. The total progeny in a branching process. J. Appl. Probab., 6: 682-686, 1969.

[12] C. Harriet Linda and G. Wiselin Jiji. Crack detection in x-ray images using fuzzy index measure. Applied Soft Computing, 11(4):3571-3579, 2011.

[13] Simon Masnou and Jean-Michel Morel. Image restoration involving connectedness. In Sixth International Workshop on Digital Image Processing and Computer Graphics, pages 84-95. International Society for Optics and Photonics, 1998.

[14] Antonio Maurício, Carlos Figueiredo, Manuel F.C. Pereira, Carlos Alves, Maïtine Bergounioux, and Olivier Rozenbaum. Assessment of stone heritage decay by x-ray computed microtomography: I-a case study of portuguese braga granite. Microscopy and Microanalysis, 21(S6):160-161, 2015.

[15] Joseph Moysan, Philippe Benoist, Gilles Corneloup, and Isabelle Magnin. Crack-like defect detection and sizing from image segmentation through co-occurrence matrix analysis. Ultrasonics, 30(6):359363, 1992.

[16] Tien Sy Nguyen, Stéphane Begot, Florent Duculty, and Manuel Avila. Free-form anisotropy: A new method for crack detection on pavement surface images. In 2011 18th IEEE International Conference on Image Processing, pages 1069-1072. IEEE, 2011.

[17] Henrique Oliveira and Paulo Lobato Correia. Automatic crack detection on road imagery using anisotropic diffusion and region linkage. In Signal Processing Conference, 2010 18th European, pages 274-278. IEEE, 2010.

[18] Mathew D. Penrose. Continuum percolation and Euclidian minimal spanning tree in high dimensions. Annal of Applied Probability, 6(2):528-544, 1996.

[19] Antony T. Popov. Approximate connectivity and mathematical morphology. In John Goutsias, Luc Vincent, and Dan S. Bloomberg, editors, Mathematical Morphology and its Applications to Image and Signal Processing, pages 149-158, Boston, MA, 2000. Springer US.

[20] AJ Pritchard, SJ Sangwine, and REN Horne. Area thresholding and silhouette extraction of simple coloured objects using hue. In Morphological and Nonlinear Image Processing Techniques, IEE Colloquium on, pages 3-1. IET, 1993.

[21] Olivier Rozenbaum, Maïtine Bergounioux, António Maurício, Carlos Figueiredo, Carlos Alves, and Luc Barbanson. Versatile three-dimensional denoising and segmentation method of x-ray tomographic images: applications to geomaterials characterizations. preprint, 2016.

[22] Zhan Shi. Branching random walks. Springer International Publishing, 2015. École d'Été de Probabilités de Saint-Flour XLII - 2012.

[23] Peggy Subirats, Jean Dumoulin, Vincent Legeay, and Dominique Barba. Automation of pavement surface crack detection using the continuous wavelet transform. In 2006 International Conference on Image Processing, pages 3037-3040. IEEE, 2006.

[24] Jay M Tenenbaum and AP Witkin. On the role of structure in vision. Human and machine vision, pages $481-543,1983$.

[25] Luc Vincent. Grayscale area openings and closings, their efficient implementation and applications. In First Workshop on Mathematical Morphology and its Applications to Signal Processing, pages 22-27, 1993.

[26] Tomoyuki Yamaguchi, Shingo Nakamura, Ryo Saegusa, and Shuji Hashimoto. Image-based crack detection for real concrete surfaces. IEEJ Transactions on Electrical and Electronic Engineering, 3(1):128-135, 2008.

[27] Song-Chun Zhu. Embedding gestalt laws in markov random fields. IEEE transactions on pattern analysis and machine intelligence, 21(11):1170-1187, 1999.

Laboratoire MAPMO, CNRS, UMR 7349, FÉdération Denis Poisson, FR 2964, Université D'OrlÉAns, B.P. 6759, 45067 OrlÉANs CEDEX 2, France

E-mail address: romain.abraham, maitine.bergounioux,pierre.debs@univ-orleans.fr 